6. Austyn, J. M. New insights into the mobilization and phagocytic activity of dendritic cells. J. Exp. Med 183, 1287-1292 (1996)

7. De Smedt, T. et al. Regulation of dendritic cell numbers and maturation by lipopolysaccharide in vivo. J. Exp. Med. 184, 1413-1424 (1996).

8. Inaba, K. et al. Generation of large numbers of dendritic cells from mouse bone marrow cultures supplemented with granulocyte/macrophage colony-stimulating factor. J. Exp. Med. 76, 1693-1702 (1992).

9. Peters, P. J., Neefjes, J. J., Oorschot, V., Ploegh, H. L. \& Geuze, H. J. Segregation of MHC class II molecules from MHC class I molecules in the Golgi complex for transport to lysosomal compartments. Nature 349, 669-676 (1991).

10. Kleijmeer, M. J. et al. MHC class II compartments and the kinetics of antigen presentation in activated mouse spleen dendritic cells. J. Immunol. 154, 5715-5724 (1995).

11. Nijman, H. W. et al. Antigen capture and major histocompatibility class II compartments of freshly isolated and cultured human blood dendritic cells. J. Exp. Med. 182, 163-174 (1995).

12. Pierre, P. et al. HLA-DM is expressed in conventional and unconventional MHC class II-containing compartments. Immunity 4, 229-239 (1996).

13. Amigorena, S., Drake, J. R., Webster, P. \& Mellman, I. Transient accumulation of new class II molecules in a novel endocytic compartment in B lymphocytes. Nature 369, 113-120 (1994).

14. Jiang, W. et al. The receptor DEC-205 expressed by dendritic cells and thymic epithelial cells is involved in antigen processing. Nature 375, 151-155 (1995).

15. Schuler, G. \& Steinman, R. M. Murine epidermal Langerhans cells mature into potent immunostimulatory dendritic cells in vitro. J. Exp. Med. 161, 526-546 (1985).

16. Larsen, C. P. et al. Migration and maturation of Langerhans cells in skin transplant and explants. J. Exp. Med. 172, 1483-1494 (1990).

17. Marsh, M. et al. Rapid analytical and preparative isolation of functional endosomes by free flow electrophoresis. J. Cell Biol. 104, 875-886 (1987).

18. Hunziker, W., Harter, C., Matter, K. \& Mellman, I. Basolateral sorting in MDCK cells requires a distinct cytoplasmic domain determinant. Cell 66, 907-920 (1991).

19. Harter, C. \& Mellman, I. Transport of the lysosomal membrane glycoprotein lgp120 (lgp-A) to lysosomes does not require appearance on the plasma membrane. J. Cell Biol. 117, 311-325 (1992)

20. Thomas, R. \& Lipsky, P. E. Could endogenous self-peptides presented by dendritic cells initiate rheumatoid arthritis? Immunol. Today 17, 559-564 (1997).

21. Cella, M., Engering, A., Pinet, V., Pieters, J. \& Lanzavecchia, A. Inflammatory stimuli induce accumulation of MHC class II complexes on dendritic cells. Nature 388, 782-787 (1997).

22. Germain, R. N. \& Hendrix, L. H. MHC class II structure, occupancy and surface expression determined by post-endoplasmic reticulum antigen binding. Nature 353, 134-139 (1991).

23. Schmid, S. L., Fuchs, R., Male, P. \& Mellman, I. Two distinct subpopulations of endosomes involved in membrane recycling and transport to lysosomes. Cell 52, 73-83 (1988).

24. Amigorena, S. et al. Invariant chain cleavage and peptide loading in post-endosomal MHC class II vesicles. J. Exp. Med. 181, 1729-1741 (1995).

25. Larsen, C. P. et al. Regulation of immunostimulatory function and costimulatory molecule (B7-1 and B7-2) expresison on murine dendritic cells. J. Immunol. 152, 5208-5219 (1994).

26. Buus, S., Colon, S., Smith, C., Freed, J. H., Miles, C. \& Grey, H. M. Interaction between a "processed" ovalbumin peptide and Ia molecules. Proc. Natl Acad. Sci. USA 83, 3968-3971 (1986).

Acknowledgements. We thank our colleagues and R. Flavell for helpful discussions, P. Webster for electron microscopy, and P. Male for confocal microscopy. P.P. was supported by a long-term EMBO fellowship, S.J.T. by an NIH predoctoral training grant, and R.M.S. and I.M. by NIH research grants.

Correspondence and requests for materials should be addressed to I.M. (e-mail: ira.mellman@yale.edu)

\section{Distinct actions of cis and trans ATP within the double ring of the chaperonin GroEL}

Hays S. Rye ${ }^{* \dagger}$ Steven G. Burston + , Wayne A. Fenton $\dagger$, Joseph M. Beechem $\ddagger$, Zhaohui Xu ${ }^{\star} \S$, Paul B. Sigler $\star_{\S}$ \& Arthur L. Horwich ${ }^{\star}$

${ }^{*}$ Howard Hughes Medical Institute, $\dagger$ Department of Genetics, School of Medicine, and $\$$ Department of Molecular Biophysics and Biochemistry, Yale University, New Haven, Connecticut 06510, USA

$\$$ Department of Molecular Physiology and Biophysics, Vanderbilt University, Nashville, Tennessee 37232, USA

The chaperonin GroEL is a double-ring structure with a central cavity in each ring that provides an environment for the efficient folding of proteins ${ }^{1-3}$ when capped by the co-chaperone GroES in the presence of adenine nucleotides ${ }^{4-8}$. Productive folding of the substrate rhodanese has been observed in cis ternary complexes, where GroES and polypeptide are bound to the same ring, formed with either ATP, ADP or non-hydrolysable ATP analogues, ${ }^{2,9}$, suggesting that the specific requirement for ATP is confined to an action in the trans ring that evicts GroES and polypeptide from the cis side 9 . We show here, however, that for the folding of malate dehydrogenase and Rubisco there is also an absolute requirement for ATP in the cis ring, as ADP and AMP-PNP are unable to promote folding. We investigated the specific roles of binding and hydrolysis of ATP in the cis and trans rings using mutant forms of GroEL that bind ATP but are defective in its hydrolysis. Binding of ATP and GroES in cis initiated productive folding inside a highly stable GroEL-ATP-GroES complex. To discharge GroES and polypeptide, ATP hydrolysis in the cis ring was required to form a GroEL-ADP-GroES complex with decreased stability, priming the cis complex for release by ATP binding (without hydrolysis) in the trans ring. These observations offer an explanation of why GroEL functions as a double-ring complex.

The monomeric protein rhodanese has recently been shown to reach native form inside cis ternary GroEL-GroES complexes that were formed in ATP, AMP-PNP or ADP, albeit at different rates $(\text { ATP }>\text { AMP-PNP }>\text { ADP })^{2,9}$. The single-ring GroEL mutant SR1 has been used to produce obligate and stable cis complexes for study ${ }^{1,2,9}$. Because it has no second ring, the SR1 mutant does not receive the signal from trans-sided ATP that normally evicts GroES and substrate ${ }^{6}$. After GroES and any of the three nucleotides were added to rhodanese-SR1 binary complexes, productive folding was shown to occur in the cis cavity. We observed that rhodanese that was refolded inside SR1-GroES formed in the presence of ATP could be released efficiently to the medium by brief incubation at $4{ }^{\circ} \mathrm{C}$, a treatment previously shown to lead to rapid dissociation of GroES from an ADP complex with GroEL $L^{6}$. Similarly, folding of ornithine transcarbamylase (OTC) from binary complexes with SR1 occurred in the presence of GroES, with almost identical kinetics with either ATP or ADP, when GroES was released by incubation at $4{ }^{\circ} \mathrm{C}$, thereby allowing OTC trimerization (data not shown). These data suggest that the previous observation of OTC folding in the presence of ATP in a single turnover from a cis ternary complex ${ }^{1}$ resulted from ADP-driven folding during preparation of the complex, with subsequent release when ATP was added. In experiments using SR1 and the green fluorescent protein (GFP), which also refolds inside cis complexes formed with any of the three nucleotides, brief treatment at $4{ }^{\circ} \mathrm{C}$ also leads to efficient release of GFP (Fig. 1a).

When the same tests were performed with two stringent substrate proteins, Rubisco from Rhodospirillum rubrum $^{10,11}$ and mitochondrial malate dehydrogenase $(\mathrm{MDH})$ from pig heart ${ }^{12-14}$, we observed that only ATP could promote reactivation from wild-type or SR1 cis ternary complexes (Fig. 1b, c). Release of GroES from SR1 by treatment at $4{ }^{\circ} \mathrm{C}$ was essential for production of enzymatic activity, because both Rubisco and $\mathrm{MDH}$ are homodimers, requiring assembly of the refolded monomeric subunits to reach active form. Direct incubation at $4{ }^{\circ} \mathrm{C}$ of metastable intermediates of Rubisco that were produced after dilution from denaturant (into the same chloride-free buffer used in all of the Rubisco studies $)^{6}$ did not result in enzymatic activity (data not shown). Remarkably, the kinetics of reactivation by the SR1-GroES ternary complexes in ATP were similar to, if not faster than, those achieved in similar reactions with wild-type GroEL, indicating that a stable foldingactive state is produced at SR1. Addition of 'trap' molecules (such as $337 / 349)^{15}$, which are able to bind but not release non-native substrate proteins, at the time of cold release of GroES had no effect on the kinetics of reactivation (kinetics not shown, but see Fig. 1f, traces 1,4$)$. These data indicate that, as with rhodanese, folding of both Rubisco and MDH proceeded to a committed state in the ATP cis ternary complexes; that is, the substrates reached conformations no longer recognizable by chaperonin.

We wished to follow directly the folding of substrate within the various cis complexes, thereby obviating any requirement for the release of GroES and peptide to assay enzymatic activity. We therefore examined by stopped-flow changes in the fluorescence total intensity and anisotropy of tryptophan residues of Rubisco in complexes formed following the addition of GroES and nucleotides to Rubisco-chaperonin binary complexes (Fig. 1d, e). This takes advantage of the absence of tryptophan from both GroEL and 
GroES, and so allows us to monitor the conformational changes occurring in the substrate during the chaperonin reaction ${ }^{16-18}$. Addition of ATP and GroES to complexes of either SR1-Rubisco or wild-type GroEL-Rubisco resulted in an initial rapid drop in total fluorescence intensity and anisotropy $\left(t_{1 / 2} \sim 1 \mathrm{~s}\right)$, followed by a steady rise in both intensity and anisotropy $\left(t_{1 / 2} \sim 3.5 \mathrm{~min}\right)$ (Fig. $1 \mathrm{~d}$, e; only anisotropy data are shown). The rate of the rising phase corresponded within a factor of two with the rate of recovery of Rubisco enzymatic activity from both SR1 and wild-type GroEL (Fig. 1b). The early decrease in anisotropy probably indicates the
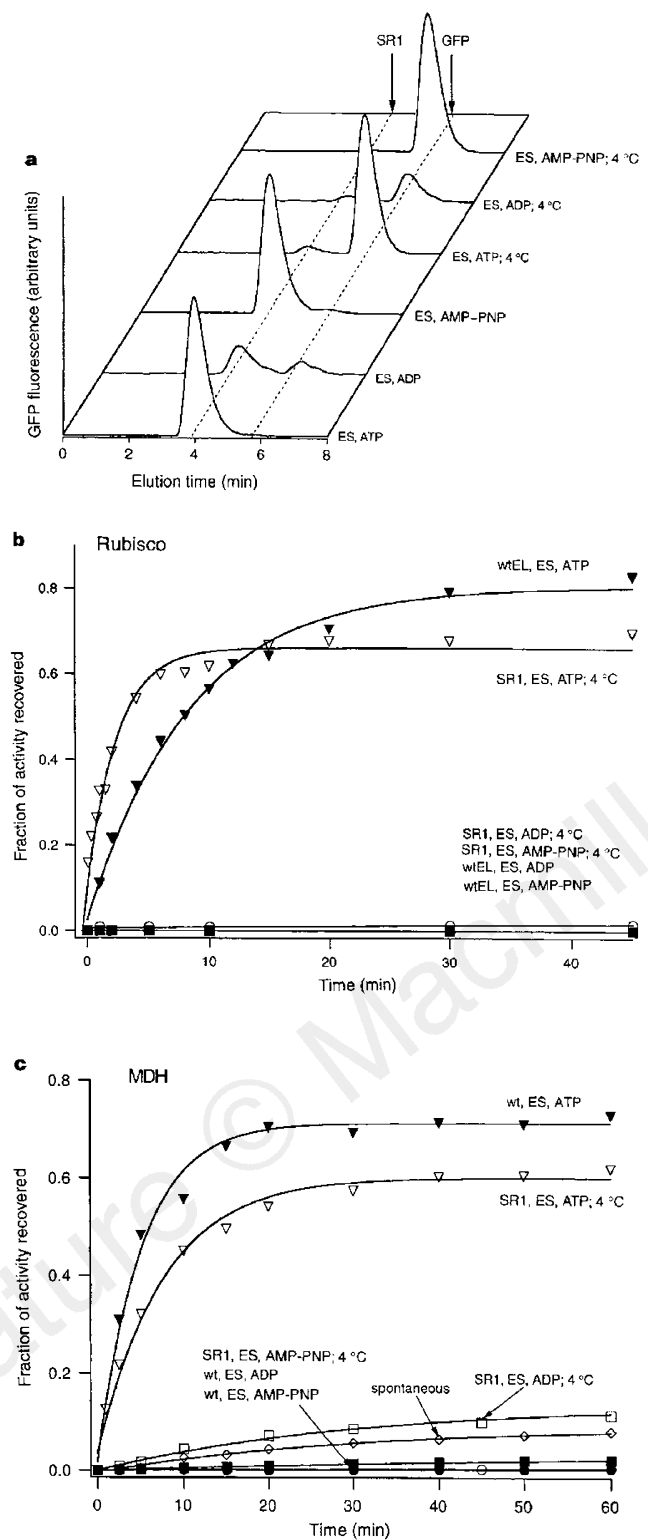
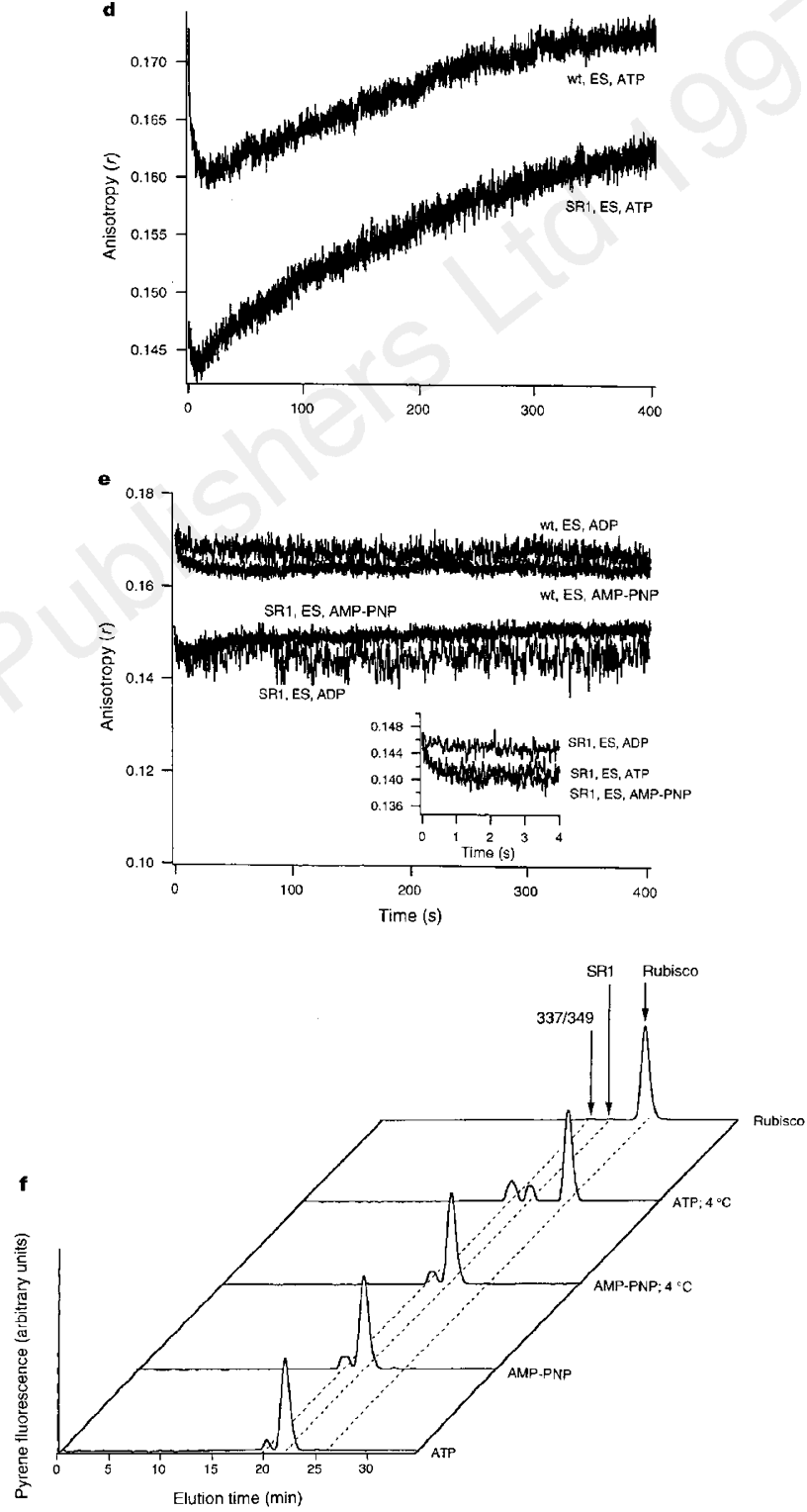

Figure 1 ATP is required in cis for the GroEL-GroES-mediated folding of two stringent substrates, Rubisco and MDH. a, Folding of the non-stringent substrate GFP inside the complex formed between the single-ring mutant, SR1, and GroES, and release of folded GFP by $4^{\circ} \mathrm{C}$ treatment. Binary complexes between acid-denatured GFP and SR1 were prepared and mixed with GroES and either ATP, ADP or AMP-PNP. Samples of the complexes, with folded, trapped GFP inside, were incubated for 10 min at either $23^{\circ} \mathrm{C}$ (unlabelled) or $4^{\circ} \mathrm{C}$, then subjected to gel filtration with on-line fluorescence detection. $\mathbf{b}$, Refolding of Rubisco by wild-type GroEL and SR1. Binary complexes were formed between acid-denatured Rubisco, and either wild-type GroEL (wtEL) or SR1, and GroES (ES) and nucleotide were added. SR1 reactions were incubated at $4^{\circ} \mathrm{C}$ for $15 \mathrm{~min}$ before enzyme assay to release bound GroES and free folded Rubisco monomers. SR 1 reactions incubated at $23^{\circ} \mathrm{C}$ demonstrated no Rubisco activity. Incubation of acid-denatured Rubisco for various times at $4{ }^{\circ} \mathrm{C}$ without chaperonin resulted in a recovery of only $1-2 \%$ activity. Total recovery is based on the activity of $100 \mathrm{nM}$ native Rubisco (monomer). c, Refolding of mitochondrial MDH by wild-type GroEL and SR1. Refolding of MDH in binary wild-type GroEL-MDH or SR1-MDH complexes was initiated by the addition of GroES and nucleotide. The spontaneous recovery of refolded MDH in the absence of chaperonins was approximately $10 \%$. Assaying SR1 reactions for enzymatic activity without prior incubation at $4{ }^{\circ} \mathrm{C}$ resulted in $<4 \%$ recovery of activity. $\mathbf{d}$, e, Folding of Rubisco with wildtype GroEL and SR1 monitored by changes in intrinsic tryptophan fluorescence anisotropy. Binary complexes were formed between acid-denatured Rubisco and either wild-type GroEL or SR1 and rapidly mixed (1:1) in a stopped-flow apparatus with solutions containing GroES and either ATP (d), ADP or AMP-PNP (e). The anisotropy of the Rubisco intrinsic tryptophan fluorescence was monitored as a function of time after mixing. Traces represent summations of 25 individual experiments (d) or $10-15$ experiments (e). Inset, early time measurements with SR1; each trace is the sum of 40-50 experiments. f, Failure of Rubisco to fold in SR1-GroES-AMP-PNP complexes correlates with inability of the polypeptide to dissociate from SR1. Binary complexes were formed between acid-denatured RUBpyr and SR1 and mixed with GroES and either ATP or AMP-PNP. After $1 \mathrm{~h}$ at $23^{\circ} \mathrm{C}, 337 / 349$ GroEL trap tetradecamer was added, and the mixture incubated at either $23^{\circ} \mathrm{C}$ or $4^{\circ} \mathrm{C}$ for an additional 10 min before gel filtration. Based on proteolytic protection experiments (not shown), GroES binds as stably to the SR1-Rubisco complex in AMP-PNP as in ATP. 

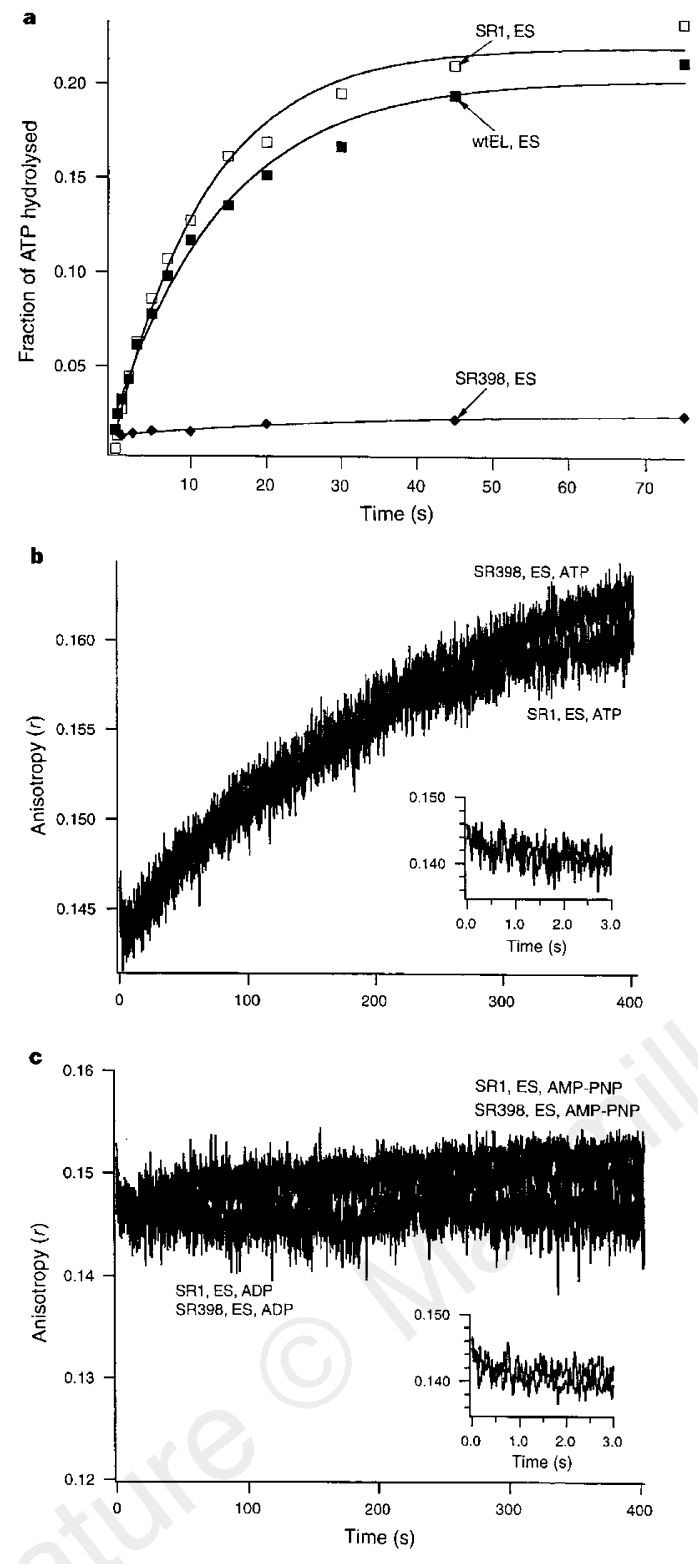

rapid mobilization of reporting regions of the Rubisco polypeptide chain, associated with release of polypeptide from the apical binding sites. The slower increase in intensity and anisotropy, associated with recovery of activity, probably reflects the burial and packing of the tryptophan side chains into the core of the refolding Rubisco monomer. In contrast, these slow rising changes were not observed when ADP or AMP-PNP was substituted for ATP, correlating with the failure of these nucleotides to support production of the native state (Fig. 1e). However, AMPPNP, but not ADP, induced the same initial rapid drop of both intensity and anisotropy that was observed with ATP (Fig. 1e, inset), suggesting that binding of GroES with AMP-PNP produces some of the same early local motion in Rubisco. However, given that no further anisotropy changes occurred (although there was a slow drop in intensity), it seems that the polypeptide is either not fully released or is recaptured by the apical peptide-binding surface $^{19}$.

The ongoing association of Rubisco with chaperonin in AMPPNP was observed directly in an experiment with SR1 and RUBpyr, which is a pyrene-labelled derivative of Rubisco. RUBpyr bears a single pyrene that does not interfere with catalytic function and allows GroEL-mediated refolding to native form, which occurs as completely as with unmodified Rubisco but at a slower rate. As

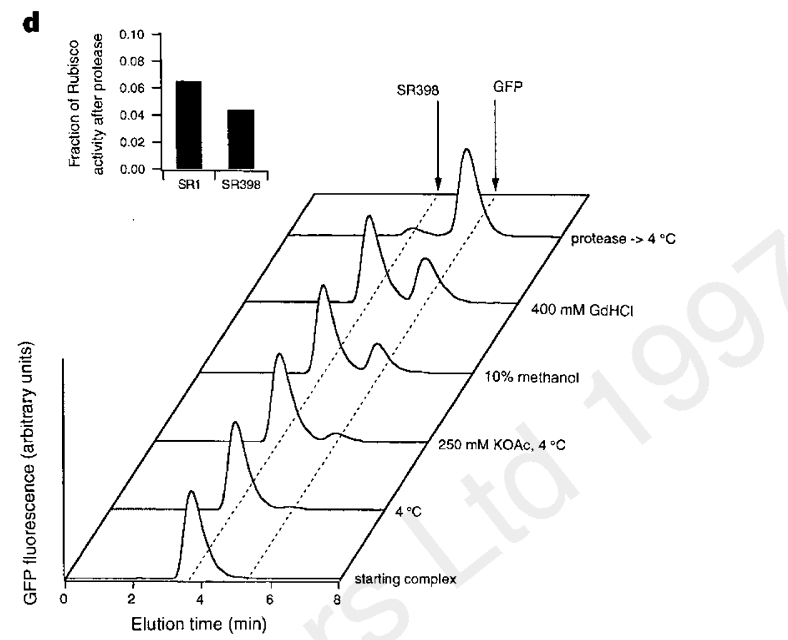

Figure 2 Binding of ATP and GroES to the cis ring of GroEL is sufficient to trigger the release and folding of Rubisco. a, ATPase activity of wild-type GroEL (wtEL), SR1 and SR398 in the presence of GroES (ES) was measured by rapid mixing/ quenching in a quench-flow apparatus. Experiments with binary complexes between each of these chaperonins and Rubisco showed no significant difference in hydrolytic activity. b, c, The SR398 ring is competent to fold Rubisco, despite absence of significant ATP hydrolytic activity. Binary complexes were formed between acid-denatured Rubisco and SR398 and rapidly mixed (1:1) in a stopped-flow apparatus with solutions containing GroES and either ATP (b), ADP (c) or AMP-PNP (c). Data for SR1 from Fig. 1 are shown again for comparison. Traces represent the summation of 25 (b) or 10-15 (c) experiments. Inset, early changes in anisotropy of the SR1 and SR398 complexes with ATP (b) and AMPPNP (c). d, High affinity of GroES for SR398 in ATP, and recovery of refolded GFP and Rubisco from SR398 ternary complexes by proteolytic treatment. GFP-SR398 binary complexes were mixed with GroES and ATP and the mixture gel filtered at $23^{\circ} \mathrm{C}$ with monitoring of fluorescence in-line. Exposure of the purified ternary complexes to $4^{\circ} \mathrm{C}$ or $250 \mathrm{mM} \mathrm{KOAc}$ at $4^{\circ} \mathrm{C}$ failed to significantly release the bound substrate. Exposure to $10 \%$ methanol or $0.4 \mathrm{M}$ guanidine- $\mathrm{HCl}(\mathrm{GdHCl})$ led to only partial release. Treatment with trypsin followed by $4{ }^{\circ} \mathrm{C}$ incubation leads to full release of GFP. For recovery of refolded Rubisco from SR398-GroES-ATP ternary complexes, a similar treatment was performed using proteinase $\mathrm{K}$ followed by assay for enzymatic activity (inset).

shown in the gel filtration profiles (Fig. 1f), RUBpyr was efficiently refolded when GroES and ATP were added to SR1-RUBpyr binary complex, because, after release with treatment at $4{ }^{\circ} \mathrm{C}$, RUBpyr migrated to the position of the Rubisco homodimer. In contrast, however, when GroES and AMP-PNP were added to the binary complex, RUBpyr comigrated with SR1 after treatment at $4{ }^{\circ} \mathrm{C}$, indicating that the non-native form remained bound to SR1, unable to be captured by added 337/349 trap (Fig. 1f). Thus both anisotropy and gel-filtration studies indicate that the formation of the cis complex in ATP releases Rubisco into the central channel, initiating folding, whereas formation of cis complex in AMP-PNP (or ADP) does not fully release the polypeptide. AMP-PNP does not seem to produce the same stereochemical changes in GroEL-GroES complexes as $\mathrm{ATP}^{20}$, reflecting the fact that AMP-PNP is not a perfect ATP homologue.

These studies demonstrated that productive conformational changes of substrate protein were triggered when ATP and GroES were added, but it was not clear whether the immediate trigger to folding was initial ATP-GroES binding or subsequent ATP hydrolysis. To resolve this question, we examined a mutant in which Asp 398 in the intermediate domain was affected, a residue shown in the GroEL-GroES-ADP crystal structure to have moved from a remote location in the unliganded structure into the equatorial 
nucleotide-binding site to a position just distal to the $\beta$-phosphate of ADP, and which contributes to the ligation of $\mathrm{Mg}^{2+}$ (ref. 19). Consistent with the structural analysis, the D398A mutant was able to bind ATP with normal affinity, but was defective in ATP turnover,
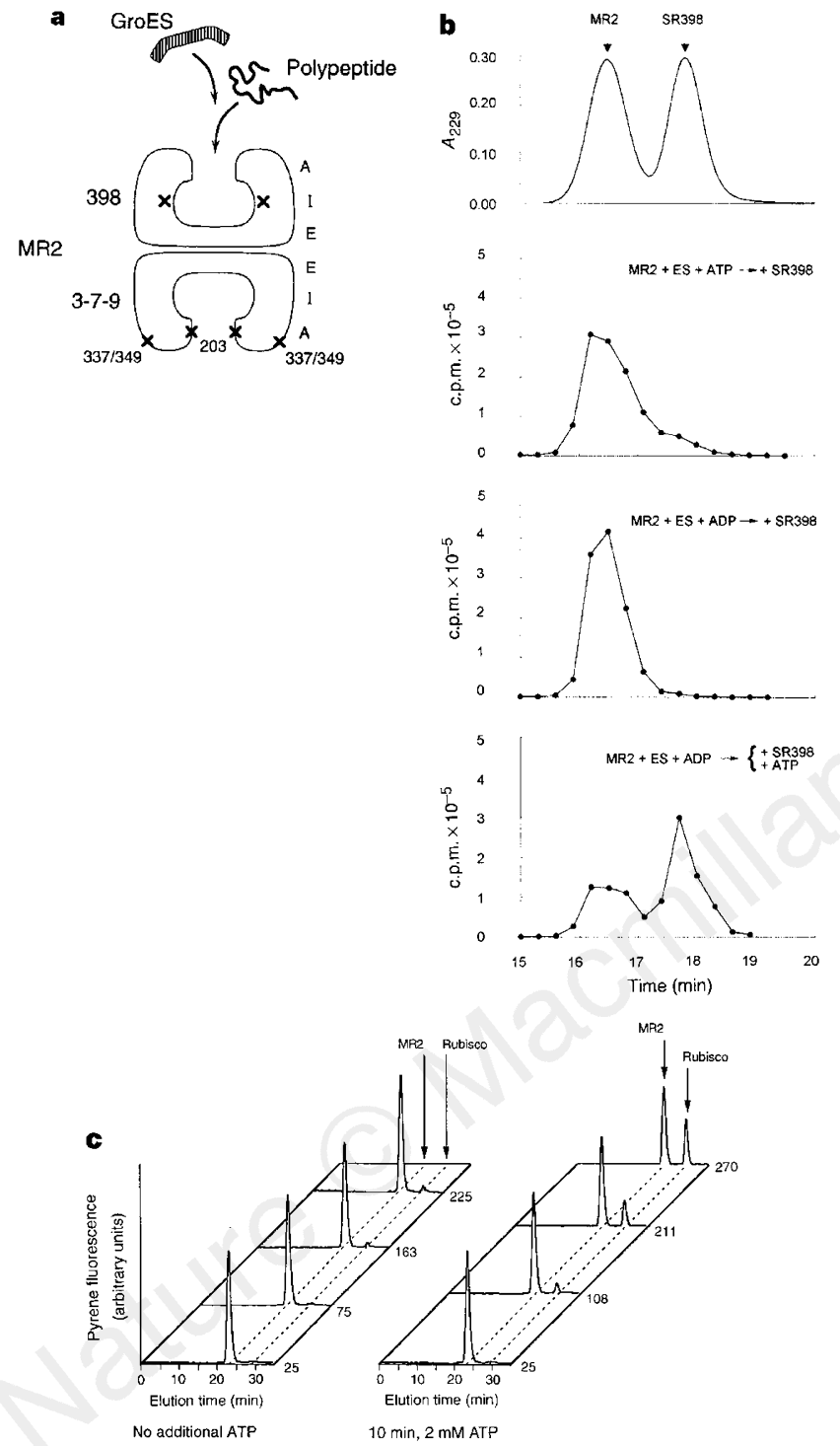

Figure 3 ATP hydrolysis in the cis ring 'primes' release of GroES and peptide ligands by trans-sided ATP. a, Schematic representation of the mixed-ring GroEL complex, MR2. The ring containing D398A mutant subunits can bind polypeptide and GroES but is defective in ATP hydrolysis; the 3-7-9 ring cannot bind polypeptide or GroES as a result of apical substitutions in its subunits, but is proficient in ATP hydrolysis ${ }^{21}$. Apical (A), intermediate (I) and equatorial (E) domains of the GroEL subunits are shown; $X$, mutations. b, ATP triggers release of GroES bound to MR2 in ADP but not in ATP. Gel filtration analyses are shown of chaperonin mixtures in which the single-ring GroES 'trap' SR398, was added to capture GroES released from binary complexes formed between MR2 and ${ }^{35} \mathrm{~S}$ radiolabelled GroES. Top, $A_{229}$ profile of MR2 and SR398; middle panels, MR2GroES complexes formed in either ATP or ADP do not release GroES. Bottom, addition of ATP triggers release of GroES from MR2-GroES binary complex formed in ADP. c, Rubisco refolded in MR2-GroES-ATP complexes can be productively released by addition of ATP after prolonged incubation of the purified ternary complexes. RUBpyr-MR2 binary complexes were incubated with GroES in the presence of ATP and the ternary complexes purified by gel filtration in the absence of nucleotide. Portions of ternary complex were exposed to ATP at the indicated time at $23^{\circ} \mathrm{C}$ after initial mixing, and mixtures were fractionated by gel filtration with in-line fluorescence detection. Migration positions of MR2 and native homodimeric Rubisco are shown. with $\sim 2 \%$ wild-type activity (data not shown). As expected, the D398A tetradecamer bound non-native rhodanese and, in the presence of GroES and ATP, formed a complex that could mediate production of native rhodanese but could not release the protein (data not shown). Similarly, when this mutation was introduced into SR1, the derived SR398 complex bound ATP and GroES in the absence of significant hydrolysis (Fig. 2a) and refolded rhodanese in the ternary complex without releasing the native protein. Fluorescencedynamics studies were also performed with SR398-Rubisco complexes. When GroES and ATP were mixed rapidly, total intensity and anisotropy changes were observed that were virtually identical to those seen with the hydrolysis-proficient SR1 complex (Fig. 2b, c). Once again, when AMP-PNP or ADP were used instead of ATP, the rising (productive) phase of anisotropy/intensity was absent, although AMP-PNP again promoted an early drop of anisotropy in SR398-bound Rubisco. We conclude that ATP-GroES binding alone is sufficient to trigger Rubisco release and productive folding in cis ternary complexes.

To determine whether Rubisco subunits could reach native form inside the SR398-GroES ternary complex, we sought to measure enzyme activity after incubation at $4^{\circ} \mathrm{C}$, as with SR1. Surprisingly, no activity was detected, owing to the failure of GroES to release from the SR398 complex. This failure was demonstrated by gel filtration of ${ }^{35}$ S-GroES-SR398-Rubisco ternary complexes that had been exposed to incubation at $4{ }^{\circ} \mathrm{C}$, where ${ }^{35} \mathrm{~S}$-GroES migrated with the complex (data not shown). This was further supported by gel filtration of SR398-GroES-GFP complexes in which, after incubation at $4{ }^{\circ} \mathrm{C}$, the fluorescent refolded GFP remained associated with SR398 (Fig. 2d). This latter analysis indicated that even transient release of GroES, which would allow escape of GFP, did not occur. Remarkably, much more severe treatments, including incubation in $0.4 \mathrm{M}$ guanidine $\mathrm{HCl}$, also failed to release GFP from SR398GroES-ATP (Fig. 2d). We finally resorted to partial proteolytic treatment with trypsin, which released intact, fluorescent GFP from the chaperonin complex (Fig. 2d). When a similar proteolytic step was performed with Rubisco bound in SR398-GroES-ATP, we observed consistent recovery of significant activity (Fig. 2d; 4-6\% in each of three independent experiments). Significantly, the same low level of recovery was observed after proteolysis of the productive SR1 ternary complex (from which $>60 \%$ activity was recovered if $4{ }^{\circ} \mathrm{C}$ release was used instead, as in Fig. $1 \mathrm{~b}$ ). The low recovery in both cases presumably results from unintended proteolytic digestion of refolded substrate protein. These findings support the indications from fluorescence studies that binding of ATP and GroES is sufficient to trigger folding of at least a fraction of Rubisco molecules to native form in the SR398 complex.

The high affinity of GroEL for GroES in ATP revealed by the study of hydrolysis-defective D398A mutants suggested that, under normal conditions, the cis ring must undergo hydrolysis of bound ATP before ATP in the trans ring can trigger GroES release. The ADP-bound state presumably comprises a lower-affinity GroELGroES interaction, or is more responsive to a signal for release of GroES produced by the trans ring. To test this, we produced a mixed-ring GroEL assembly ${ }^{21}$, composed of a D398A ring, which was able to bind polypeptide and GroES but unable to hydrolyse ATP, and a 203/337/349 (3-7-9) ring, which was unable to bind substrate or GroES but able to provide normal ATP function in trans to the D398A ring (see Fig. 3a). The mixed-ring complex, MR2, was tested for ability to stably bind ${ }^{35} \mathrm{~S}$-labelled GroES in ATP or ADP. In the presence of ATP, MR2 stably bound GroES, failing to release it even though there was hydrolysis in the 3-7-9 ring (at a rate $\sim 21 \%$ of wild-type, unaffected by GroES). In particular, when SR398 was added as a GroES 'trap' to a mixture containing MR2-GroES and ATP, no transfer of radiolabelled GroES from MR2 to SR398 was observed by gel-filtration analysis (Fig. 3b). Thus the high affinity for GroES of the D398A ring of MR2 in ATP seems to recapitulate that observed with SR398 in ATP. GroES was also stably bound to 
MR2 in ADP, although if ADP was absent from the gel filtration running buffer, the GroES was released (data not shown). This would seem to be consistent with an apparently lower affinity interaction of GroES with the D398A ring in ADP (compared with no release from the ATP complex when it was gel filtered in the absence of nucleotide). More importantly, however, when the MR2-GroES complex formed in ADP was exposed to ATP, GroES was released efficiently, and was detected through its capture by coincubated SR398 (Fig. 3b). This suggests that the pre-bound ADP cis complex emulates the post-hydrolysis ADP cis complex in being similarly coupled to the action of trans-sided ATP, allowing GroES to be released.

The consequences of cis ATP hydrolysis for release of folded polypeptide were examined more directly by forming RUBpyrMR2-GroES complexes in ATP, purifying them by gel filtration, and incubating them in the absence of nucleotide for prolonged periods, during which the bound ATP slowly hydrolysed in the D398A ring. When such complexes were then exposed to ATP and the reaction mixtures gel filtered, there was a progressive increase with incubation time in recovery of fluorescence at the position of native homodimeric Rubisco, reflecting the gradual priming of the cis ring for trans-ATP-driven release of the folded RUBpyr (Fig. 3c).

Once we had observed the specific requirement for binding of ATP in the cis ring to promote folding, with AMP-PNP unable to substitute, we then investigated whether a similar requirement might be operative in the trans ring with respect to driving GroES/polypeptide release from the cis side. Previous studies have observed that AMP-PNP and ATP- $\gamma S$ supplied in trans are unable to support the release of GroES from cis, but have been interpreted to indicate that hydrolysis is required in trans for eviction from $\mathrm{cis}^{6}$. To address this question, the hydrolysis-defective D398A tetradecamer was used to form folding-active cis ternary complexes in ATP which were allowed to slowly hydrolyse bound cis ATP over $2 \mathrm{~h}$ to produce a 'primed' cis-ADP state (as in Fig. 3c). The complexes were then incubated briefly with ADP, AMP-PNP or ATP. When GFP was examined as a substrate, gel-filtration studies showed that the folded protein remained stably associated in D398A ternary com- plexes (purified away from the nucleotide) over the 2-h time period. GFP was not released upon subsequent addition of ADP or AMP-PNP (Fig. 4). However, when ATP was added, gel filtration after 2 min showed that $>60 \%$ of the GFP was released (Fig. 4), which corresponds to the efficiency of GFP release observed when ATP was added to a wild-type cis-ADP-GFP complex (Fig. 4). $\mathrm{MDH}$ was also used as a substrate in a similar experiment (not shown), in which limiting amounts (just sufficient for formation of cis complex) of ATP were supplied, followed by incubation for $2 \mathrm{~h}$. Once again, brief exposure to ATP but not ADP or AMP-PNP produced recovery of enzymatic activity, the apparent result of release of refolded monomer from the cis ternary ADP complex only by ATP. These studies with D398A indicate that binding of ATP in trans, independent of hydrolysis, is sufficient to promote the release of GroES and polypeptide from cis-ADP complexes.

In summary, our results indicate that GroEL-mediated folding of stringent substrates like Rubisco and $\mathrm{MDH}$ requires action of ATP in both the cis and trans rings (Fig. 5). In the cis ring, binding of ATP and GroES triggers folding, potentially beginning within $1 \mathrm{~s}$, a finding that is in accord with the early and rapid drop of anisotropy reported here and elsewhere ${ }^{2}$. ATP, specifically, promotes the complete release of stringent substrates like Rubisco into the central channel, which enlarges at least twofold in volume in association with GroES binding and changes in surface character from hydrophobic to hydrophilic ${ }^{19}$ (Fig. 1d, f). Hydrolysis of ATP in the cis ring, occurring with a $t_{1 / 2}$ of $6-8 \mathrm{~s}$ (see Fig. $2 \mathrm{a}$ and ref. 7 ), is then necessary to relax the high-affinity interaction of GroES with ATP-bound GroEL, priming the ring for the release of GroES, which is accomplished by subsequent binding of ATP in the trans ring.

\section{Methods}

Proteins. Tetradecameric GroEL with the D398A substitution (D398A) was produced by polymerase chain reaction (PCR)-mediated site-directed mutagenesis of a plasmid encoding wild-type GroEL ${ }^{21}$. A single-ring mutant containing the D398A substitution (SR398) was produced by exchanging a

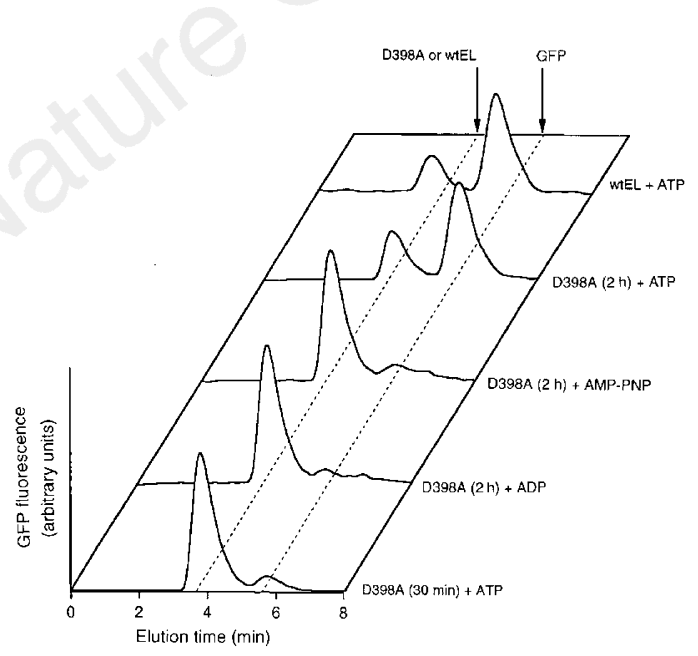

Figure 4 Release of GFP refolded in tetradecameric D398A cis ternary complexes by subsequent addition in trans of ATP but not ADP or AMP-PNP. GFP-D398A binary complexes were incubated with GroES and ATP, treated briefly with proteinase $\mathrm{K}$ to remove any GFP bound in trans, then purified by gel filtration in the absence of nucleotide. The complexes were then incubated for $30 \mathrm{~min}$ or $2 \mathrm{~h}$, then exposed to ATP, ADP or AMP-PNP for 2 min and rapidly gel filtered with inline fluorescence detection. By $2 \mathrm{~h}$ the amount of GFP released by ATP from D398A tetradecamer is similar to that obtained from GFP-wtGroEL-GroES-ADP cis complexes exposed to ATP for 1 min. ATP triggered release from D398A, but ADP and AMP-PNP had no effect.

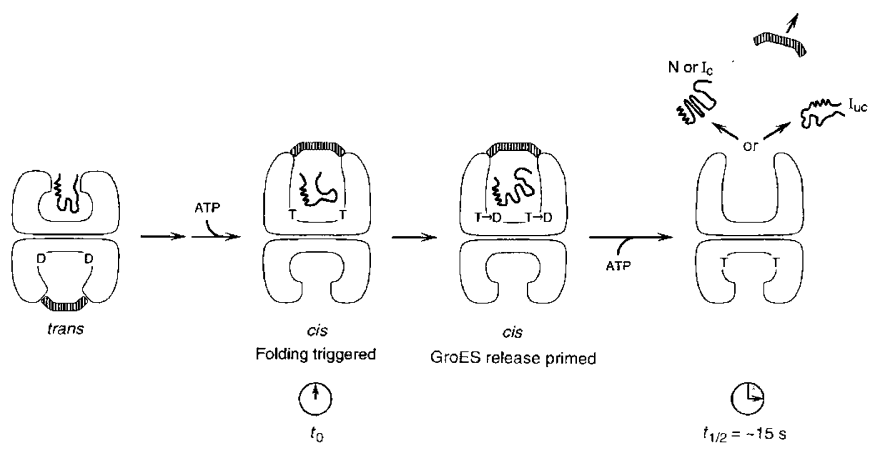

Figure 5 Schematic representation of the action of ATP in cis and trans rings during GroEL-GroES-mediated protein folding. Polypeptide is initially bound in the trans ring of a GroEL-GroES binary complex, and a step of ATP-triggered GroES release and rebinding or of binding of a second GroES to the polypeptidebound ring occurs, forming a cis complex in which polypeptide is sequestered in the GroEL central channel underneath GroES ${ }^{23,24}$. The act of formation of the cis complex, through binding of ATP and GroES to the ring with bound polypeptide, triggers rapid release of polypeptide from the apical binding sites into the central channel, and folding begins $\left(t_{0}\right)$ (see Figs 1,2 ). Hydrolysis of ATP in the cis ring weakens the high-affinity interaction between GroES and (cis)ATP-bound GroEL (see Fig. 3), and binding of ATP in trans subsequently produces release of GroES and polypeptide from the cis ring ( $t_{1 / 2} \sim 15 \mathrm{~s}$ ) (see Fig. 4). D, ADP; T, ATP; T $\rightarrow \mathrm{D}$, hydrolysis of ATP; N, native; $I_{c}$, non-native conformation committed to completing folding to native form (no longer recognizable by chaperonin); Iuc, non-native form that must be rebound by chaperonin or by a different chaperone to reach native form. 
restriction fragment with coding sequences bearing the D398A mutation for the wild-type segment with that in an expression plasmid encoding SR1. GroEL, SR1 and the derivatives, as well as GroES, were expressed in Escherichia coli and purified as described ${ }^{1}$. Metabolically labelled $\left[{ }^{35} \mathrm{~S}\right] \mathrm{GroES}$ was produced as described ${ }^{1}$. Pig heart mitochondrial malate dehydrogenase (Sigma) was purified by gel filtration chromatography. Soluble, fluorescent GFP and Rubisco from Rhodospirillum rubrum were expressed at $20^{\circ} \mathrm{C}$ from $\mathrm{T} 7$ promoters in BL21 transformants in the absence of induction and were purified by ion-exchange chromatography.

GFP refolding. All GFP folding experiments were conducted in buffer A (50 mM HEPES, pH 7.6, $5 \mathrm{mM} \mathrm{KOAc,} 10 \mathrm{mM} \mathrm{Mg}(\mathrm{OAc})_{2}, 1 \mathrm{mM} \mathrm{DTT}$ ) at $23^{\circ} \mathrm{C}$. GFP $(\sim 530 \mu \mathrm{M})$ was acid-denatured by a 5 -fold dilution with $25 \mathrm{mM}$ glycinephosphate buffer ( $\mathrm{pH} 2.0$ ). After $5 \mathrm{~min}$ at $23^{\circ} \mathrm{C}$, binary complexes were generated by diluting the denatured GFP 11 -fold $(9 \mu \mathrm{M}$ final concentration) into $27 \mu \mathrm{M}$ SR1 (Fig. 1a) or SR398 (Fig. 2d) in buffer A and incubating for $10 \mathrm{~min}$ at $23^{\circ} \mathrm{C}$. For SR1 experiments (Fig. 1a), GroES was added to $50 \mu \mathrm{M}$ and either ATP to $1.5 \mathrm{mM}$ or AMP-PNP to $5 \mathrm{mM}$, or GroES was added to $165 \mu \mathrm{M}$ and ADP to $1.5 \mathrm{mM}$. Folding mixtures were incubated at $23^{\circ} \mathrm{C}$ for $45 \mathrm{~min}$. One portion was placed at $4^{\circ} \mathrm{C}$ for $10 \mathrm{~min}$, while another was maintained at $23^{\circ} \mathrm{C}$. All samples were then chromatographed over three Tosohaas TSK-gel guard columns connected in series. In-line fluorescence detection of renatured GFP used excitation at $339 \mathrm{~nm}$ and an emission cut-off filter centred at $495 \mathrm{~nm}$. For SR398 experiments (Fig. 2d), GroES was added to $66 \mu \mathrm{M}$ and ATP to $2 \mathrm{mM}$, and the mixture was incubated at $23^{\circ} \mathrm{C}$ for $15 \mathrm{~min}$, then gel filtered on the guard columns. The purified ternary complex was either reinjected or first treated for $15 \mathrm{~min}$ with the indicated conditions. For protease treatment, one portion was supplemented with trypsin to $15 \mu \mathrm{g} \mathrm{ml}^{-1}$ and incubated at $23^{\circ} \mathrm{C}$ for $10 \mathrm{~min}$. Soybean trypsin inhibitor was then added to $125 \mu \mathrm{g} \mathrm{ml}^{-1}$, the sample incubated at $4{ }^{\circ} \mathrm{C}$ for $10 \mathrm{~min}$, and then injected. For D398A (tetradecamer) studies (Fig. 4), ternary complex was formed as above. After $15 \mathrm{~min}$ at $23^{\circ} \mathrm{C}$, proteinase $\mathrm{K}$ was added $\left(1 \mu \mathrm{g} \mathrm{ml}^{-1}\right.$ final concentration) to remove GFP bound in trans. After $5 \mathrm{~min}$, PMSF was added to $1 \mathrm{mM}$ and the complex was purified by gel filtration on the tandem guard columns in buffer A. The purified complex was incubated in the dark at $23^{\circ} \mathrm{C}$ for $30 \mathrm{~min}$ or $2 \mathrm{~h}$. Samples were then supplemented with either ATP $(2 \mathrm{mM})$, ADP $(5 \mathrm{mM})$ or AMP-PNP $(5 \mathrm{mM})$, incubated for $2 \mathrm{~min}$, then reinjected to the guard columns and analysed by on-line fluorescence detection. Wild-type GroELGroES-GFP complex was prepared similarly, except that ADP $(1 \mathrm{mM})$ and GroES $150 \mu \mathrm{M}$ were used. Despite the low yield of this assembly, the ADPternary complex was stable until challenged with ATP, as determined by rechromatographing.

Rubisco refolding. Rubisco was denatured by a 1:55 dilution into $25 \mathrm{mM}$ glycine-phosphate buffer, pH 2.0. Immediately after a 3-5 min incubation at $23^{\circ} \mathrm{C}$, binary complexes were formed by mixing the unfolded Rubisco with chaperonin in a total volume of $1.5 \mathrm{ml}$ buffer $\mathrm{A}$. The final concentrations were $130 \mathrm{nM}$ wild-type GroEL, $300 \mathrm{nM}$ SR1 or SR398, and $100 \mathrm{nM}$ Rubisco (monomer). Folding was initiated by addition of a two-fold molar excess of GroES (over chaperonin) and either ATP $(1 \mathrm{mM})$, ADP $(5 \mathrm{mM})$ or AMP-PNP $(5 \mathrm{mM})$ (final concentrations). For wild-type reactions, at the times indicated, ATP was removed by adding hexokinase and glucose (final concentrations, $0.08 \mathrm{U}^{-1} \mathrm{l}^{-1}$ hexokinase and $10 \mathrm{mM}$ glucose). For SR1 reactions, ATP was removed $30 \mathrm{~s}$ after its addition by adding hexokinase and glucose. SR1 reactions were incubated at $4{ }^{\circ} \mathrm{C}$ for $15 \mathrm{~min}$ before enzyme assay to release bound GroES and free folded Rubisco monomers. SR1 samples were briefly allowed to re-equilibrate to $23^{\circ} \mathrm{C}$ before enzyme assay. For proteolytic release of Rubisco from SR398-GroES and SR1-GroES ternary complexes (Fig. 2d), after $7 \mathrm{~min}$ of refolding at $23^{\circ} \mathrm{C}$ (see Fig. 1b) the mixture was supplemented to $3 \mathrm{mM} \mathrm{Ca}(\mathrm{OAc})_{2}$ and proteinase $\mathrm{K}$ was added to $125 \mu \mathrm{g} \mathrm{ml}^{-1}$. After incubation at $23^{\circ} \mathrm{C}$ for $6 \mathrm{~min}$, PMSF was added to $3 \mathrm{mM}$ and the mixture assayed after a further $10 \mathrm{~min}$ at $23^{\circ} \mathrm{C}$ for Rubisco activity. Rubisco enzyme activity was determined by incorporation of $\left[{ }^{14} \mathrm{C}\right] \mathrm{CO}_{2}$ into acid-precipitable products ${ }^{10}$.

MDH refolding. All MDH refolding studies were performed in $50 \mathrm{mM}$ Tris$\mathrm{HCl}, \mathrm{pH} 7.4,50 \mathrm{mM} \mathrm{KCl}, 10 \mathrm{mM} \mathrm{MgCl}, 10 \mathrm{mM}$ DTT (buffer B). MDH was denatured for $1 \mathrm{~h}$ in $5 \mathrm{M}$ urea in buffer $\mathrm{B}$ at $30^{\circ} \mathrm{C}$. Binary complexes were formed between wild-type GroEL or SR1 and MDH by rapidly diluting the denatured MDH 250 -fold using a solution of $1.2 \mu \mathrm{M}$ wild-type GroEL or $5 \mu \mathrm{M}$
SR1 in buffer B such that the final concentration of MDH was $1 \mu \mathrm{M}$ with respect to subunits. Refolding was initiated by the addition of a 3-fold molar excess of GroES and either $2 \mathrm{mM}$ ATP, $10 \mathrm{mM}$ ADP or $10 \mathrm{mM}$ AMP-PNP, followed by incubation at $30^{\circ} \mathrm{C}$. At various times a portion was withdrawn and, in the case of wild-type GroEL, assayed immediately for enzymatic activity. In the case of the SR1-mediated reaction, the ATP was removed by hexokinase and glucose as before, then incubated at $4{ }^{\circ} \mathrm{C}$ for $10-15 \mathrm{~min}$ before assay to release bound GroES and free folded, assembly-competent $\mathrm{MDH}$ monomers. For the experiment with D398A, binary complex was formed with $5 \mu \mathrm{M}$ MDH subunit and $7 \mu \mathrm{M}$ D398A oligomer (100 $\mu \mathrm{M}$ subunit). GroES and ATP were added to $10 \mu \mathrm{M}$ and $50 \mu \mathrm{M}$ final concentrations, respectively. After $2 \mathrm{~h}$ at $30^{\circ} \mathrm{C}$, proteinase $\mathrm{K}$ was added at $800 \mathrm{ng} \mathrm{ml}^{-1}$, and incubation carried further for $5 \mathrm{~min}$. PMSF was then added to $1 \mathrm{mM}$. Samples were then supplemented with ATP, AMP-PNP or ADP to $4 \mathrm{mM}$ and after $90 \mathrm{~s}$ were assayed for $\mathrm{MDH}$ activity. MDH enzyme assay was performed by diluting a portion from the folding assay into $1 \mathrm{mM}$ ketomalonate, $0.2 \mathrm{mM} \mathrm{NADH}$, $10 \mathrm{mM}$ DTT, and $50 \mathrm{mM}$ Tris- $\mathrm{HCl}, \mathrm{pH} 7.4$, and following the oxidation of $\mathrm{NADH}$ at $340 \mathrm{~nm}$ (ref. 14).

Stopped-flow fluorescence anisotropy. The stopped-flow fluorescence anisotropy apparatus used in these studies was constructed by H.S.R. using as a template a design from the laboratory of J. Beechem ${ }^{16}$. It consists of a BioLogic SFM/4 stopped-flow head coupled to an excitation source and monochromator (Photon Technologies International) through a fibre-optic bundle designed to provide a small slit-shaped $(3 \times 0.66 \mathrm{~mm})$ excitation beam to the stopped-flow cuvette (FC.15). The two emission channels are collected in Tformat through a pair of non-fibre, large core diameter, high numerical aperature (NA 0.59) light guides (Oriel) placed directly against the stoppedflow cuvette to maximize light collection efficiency. Polarization selection of the excitation and emission used film polarizers (Meadowlark Optics) placed over the end of each light guide at the stopped-flow cuvette. The collected fluorescence is spectrally filtered and detected with a pair of Hammamatsu PMTs (R4457P) operated in photon-counting mode. Data collection was as previously described ${ }^{16}$. For stopped-flow experiments involving Rubisco, a sample was diluted $(1: 9)$ with $25 \mathrm{mM}$ glycine-phosphate buffer, $\mathrm{pH} 2.0$, to produce a $15.6 \mu \mathrm{M}$ acid-denatured Rubisco stock. Each binary complex was generated by diluting $480 \mu \mathrm{l}$ of this stock into $5 \mathrm{ml}$ of buffer A containing either wild-type GroEL $(2 \mu \mathrm{M})$, SR1 $(4 \mu \mathrm{M})$ or SR398 $(4 \mu \mathrm{M})$. After $10 \mathrm{~min}$ at $23^{\circ} \mathrm{C}$, the binary mixture was loaded into one syringe of the stopped-flow apparatus. The binary mixture was rapidly mixed $(1: 1)$ in the stopped-flow (at $23^{\circ} \mathrm{C}$ ) with a solution containing GroES $(6 \mu \mathrm{M})$ and either ATP $(2 \mathrm{mM})$, ADP (10 $\mathrm{mM})$ or AMP-PNP $(10 \mathrm{mM})$.

Quenched-flow ATPase assay. ATPase activities of wild-type GroEL, SR1 and SR398 were determined using a quenched-flow rapid mixing apparatus (KinTek) at $20^{\circ} \mathrm{C}$. Equal volumes $(15 \mu \mathrm{l})$ of chaperonin in buffer A and GroES/ $\left[\gamma^{32} \mathrm{P}\right]$ ATP in buffer A were mixed to give final concentrations of $2.85 \mu \mathrm{M}$ chaperonin, $6 \mu \mathrm{M}$ GroES, $100 \mu \mathrm{M}\left[\gamma^{-}{ }^{32} \mathrm{P}\right]$ ATP $\left(300 \mu \mathrm{Ci} \mu \mathrm{mol}^{-1}\right)$, then the reaction was quenched with $67 \mu \mathrm{l} 1.5 \mathrm{M}$ formic acid/1.5 mM potassium phosphate at the times indicated. Note that the ATP concentration is such that hydrolysis by wild type is not at steady state after one turnover. Portions of each sample were chromatographed on polyethylene imine thin-layer plates, developed with $1 \mathrm{M}$ formic acid and $0.25 \mathrm{M} \mathrm{LiCl}$. Radioactivity in phosphate and ATP regions was quantified by phosphorimager analysis.

Labelling of native Rubisco with pyrene (RUBpyr). Rubisco $\left(0.8 \mathrm{mg} \mathrm{m}^{-1}\right.$ in $100 \mathrm{mM}$ sodium phosphate, $\mathrm{pH} 7.6$ ) was reduced with $2 \mathrm{mM}$ TCEP and labelled with a 6-7-fold excess of $N$-1-pyrene-maleimide, added in two portions. The reaction was quenched by adding glutathione $(2 \mathrm{mM})$, and the labelled Rubisco was separated from unbound dye by gel filtration (PD-10 column, Pharmacia). The labelling ratio was $1: 1$, pyrene to Rubisco monomer, as determined by absorption spectroscopy, using extinction coefficients of $67,000 \mathrm{M}^{-1} \mathrm{~cm}^{-1}$ at $280 \mathrm{~nm}$ for the Rubisco monomer ${ }^{22}$ and $36,000 \mathrm{M}^{-1} \mathrm{~cm}^{-1}$ at $340 \mathrm{~nm}$ for reacted $\mathrm{N}$-1-pyrene-maleimide (Molecular Probes). Labelling of a single cysteine (usually the surface-accessible Cys 58) was indicated by detection of a single major tryptic peptide with strong absorption at $340 \mathrm{~nm}$ during separation by $\mathrm{C} 8$ reverse-phase chromatography. The specific activity of the labelled enzyme was indistinguishable from that of unlabelled Rubisco, and refolding of acid-denatured RUBpyr was dependent upon GroEL, GroES and 
ATP, although renaturation occurred at a rate approximately half that of the unlabelled enzyme.

Refolding and gel filtration with RUBpyr. For RUBpyr refolding, the labelled enzyme was denatured in acid glycine-phosphate buffer as described above. For the experiment shown in Fig. 1, binary complexes were formed by incubating denatured RUBpyr with SR1 in buffer A (final concentrations, $2 \mu \mathrm{M}$ RUBpyr and $4 \mu \mathrm{M}$ SR1) for at least $10 \mathrm{~min}$ at $23^{\circ} \mathrm{C}$. GroES was added to $5 \mu \mathrm{M}$, and folding was initiated by adding either ATP to $1 \mathrm{mM}$ or AMP-PNP to $5 \mathrm{mM}$. After $1 \mathrm{~h}$ at $23^{\circ} \mathrm{C}, 337 / 349$ GroEL trap was added to $6.5 \mu \mathrm{M}$ and incubation continued at either $23^{\circ} \mathrm{C}$ or $4^{\circ} \mathrm{C}$ for a further $15 \mathrm{~min}$. All samples were then subjected to gel filtration on a TSK G-4000SW $\mathrm{xl}_{\mathrm{xl}}$ column equilibrated in buffer A. For the experiment shown in Fig. 3, a 20- $\mu$ M solution of unfolded RUBpyr was mixed with buffer A containing MR2 to give final concentrations of $4 \mu \mathrm{M}$ RUBpyr and $5 \mu \mathrm{M}$ MR2. After $10 \mathrm{~min}$ at $23^{\circ} \mathrm{C}$, GroES was added to $10 \mu \mathrm{M}$ and ATP to $2 \mathrm{mM}$, and the mixture incubated at $23^{\circ} \mathrm{C}$ for $5 \mathrm{~min}$. Unbound ATP was removed by rapid $(4 \mathrm{~min})$ gel filtration over three TSK guard columns connected in series and equilibrated in buffer A. The purified MR2-RUBpyrGroES complex was incubated at $23^{\circ} \mathrm{C}$ for the times indicated, then $200-\mu \mathrm{l}$ portions of the complex were either directly injected onto the G-4000 gel filtration column equilibrated in buffer A (to assess the intrinsic stability of the complex), or were supplemented with ATP to $2 \mathrm{mM}$ for $10 \mathrm{~min}$, then gel filtered. For all experiments, pyrene fluorescence was detected with an in-line fluorescence detector with excitation at $339 \mathrm{~nm}$ and an emission long-pass filter centred at $470 \mathrm{~nm}$.

Production of mixed-ring complex and GroES binding. Mixed-ring complex MR2 was produced and purified as described previously for MR1 (ref. 21), with incubation for at least $1 \mathrm{~h}$ of the parent tetradecamers, D398A and 3-7-9 in MgATP at $42{ }^{\circ} \mathrm{C}$. For studies of GroES binding to MR2, ${ }^{35} \mathrm{~S}$-GroES (40,000 c.p.m. pmol ${ }^{-1}$ ) was incubated at a final concentration of $0.3 \mu \mathrm{M}$ with $1 \mu \mathrm{M}$ MR2 and either ATP or ADP ( $1 \mathrm{mM})$ in $10 \mathrm{mM} \mathrm{KCl}, 25 \mathrm{mM}$ Tris- $\mathrm{HCl}, \mathrm{pH}$ $7.4,1 \mathrm{mM} \mathrm{MgCl}_{2}$. After $20 \mathrm{~min}$ at $20^{\circ} \mathrm{C}$, SR398 was added to $2 \mu \mathrm{M}$ and, where indicated, ATP was added to a final concentration of $10 \mathrm{mM}$. After $5 \mathrm{~min}$ further incubation, the mixtures were chromatographed on a $\mathrm{G} 4000 \mathrm{SW}_{\mathrm{xl}}$ column in the same buffer containing $1 \mathrm{mM}$ ADP. Fractions were collected and counted directly.

Received 22 May; accepted 7 July 1997.

1. Weissman, J. S. et al. Mechanism of GroEL action: productive release of polypeptide from sequestered position under GroES. Cell 83, 577-588 (1995).

2. Weissman, J. S., Rye, H. S., Fenton, W. A., Beechem, J. M. \& Horwich, A. L. Characterization of the active intermediate of a GroEL-GroES-mediated protein folding reaction. Cell 84, 481-490 (1996).

3. Mayhew, M. et al. Protein folding in the central cavity of the GroEL-GroES chaperonin complex. Nature 379, 420-426 (1996).

4. Chandrasekhar, G. N., Tilly, K., Woolford, C., Hendrix, R. \& Georgopoulos, C. Purification an properties of the GroES morphogenetic protein of Escherichia coli. J. Biol. Chem. 261, 12414-12419 (1986).

Jackson, G. S. et al. Binding and hydrolysis of nucleotides in the chaperonin catalytic cycle: implications for the mechanism of assisted protein folding. Biochemistry 32, 2554-2563 (1993).

6. Todd, M. J., Viitanen, P. \& Lorimer, G. H. Dynamics of the chaperonin ATPase cycle: implications for facilitated protein folding. Science 265, 659-666 (1994)

7 Burston, S. G., Ranson, N. A. \& Clarke, A. R. The origins and consequences of asymmetry in the chaperonin reaction cycle. J. Mol. Biol. 249, 138-152 (1995).

8. Hayer-Hartl, M., Martin, J. \& Hartl, F.-U. Asymmetrical interaction of GroEL and GroES in the ATPase cycle of assisted protein folding. Science 269, 836-841 (1995)

9. Hayer-Hartl, M. K., Weber, F. \& Hartl, F.-U. Mechanism of chaperonin action: GroES binding and release can drive GroEL-mediated protein folding in the absence of ATP hydrolysis. EMBO J. 15, 6111-6121 (1996)

10. Goloubinoff, P., Christeller, J. T., Gatenby, A. A. \& Lorimer, G. H. Reconstitution of active dimeric ribulose bisphosphate carboxylase from an unfolded state depends on two chaperonin proteins and Mg-ATP. Nature 342, 884-889 (1989).

11. Todd, M. J., Lorimer, G. H. \& Thirumalai, D. Chaperonin-facilitated protein folding: Optimization of rate and yield by an iterative annealing mechanism. Proc. Natl Acad. Sci. USA 93, 4030-4035 (1996).

12. Ranson, N. A., Dunster, N. J., Burston, S. G. \& Clarke, A. R. Chaperonins can catalyze the reversal of early aggregation steps when a protein misfolds. J. Mol. Biol. 250, 581-586 (1995)

13. Peralta, D., Hartman, D. J., Hoogenraad, N. J. \& Hoj, P. B. Generation of a stable folding intermediate which can be rescued by the chaperonins GroEL and GroES. FEBS Lett. 339, 45-49 (1994).

14. Ranson, N. A., Burston, S. G. \& Clarke, A. R. Binding, encapsulation and ejection: substrate dynamics during a chaperonin-assisted folding reaction. J. Mol. Biol. 266, 656-664 (1997).

15. Weissman, J. S., Kashi, Y., Fenton, W. A. \& Horwich, A. L. GroEL-mediated protein folding proceeds by multiple rounds of binding and release of nonnative forms. Cell 78, 693-702 (1994).

16. Otto, M. R., Lillo, M. P. \& Beechem, J. M. Resolution of multiphasic reactions by the combination of fluorescence total-intensity and anisotropy stopped-flow kinetic experiments. Biophys. J. 67, 25112521 (1994)

17. Beechem, J. M., Sherman, M. A. \& Mas, M. T. Sequential domain unfolding in phosphoglycerate kinase: fluorescence intensity and anisotropy stopped-flow kinetics of several tryptophan mutants. Biochemistry 34, 13943-13948 (1995).

18. Etfink, M. R. The use of fluorescence methods to monitor unfolding transitions in proteins. Biophys. J. 66, 482-501 (1994)
19. Xu, Z., Horwich, A. L. \& Sigler, P. B. The crystal structure of the asymmetric GroEL-GroES-(ADP) chaperonin complex. Nature 388, 741-750 (1997)

20. Roseman, A. M., Chen, S., White, H., Braig, K. \& Saibil, H. R. The chaperonin ATPase cycle: mechanism of allosteric switching and movements of substrate-binding domains in GroEL. Cell 87, 241-251 (1996).

21. Burston, S. G., Weissman, J. S., Farr, G. W., Fenton, W. A. \& Horwich, A. L. Release of both native and non-native proteins from a cis-only GroEL ternary complex. Nature 383, 96-99 (1996).

22. Pierce, J. \& Reddy, G. S. The sites for catalysis and activation of ribulosebisphosphate carboxylase share a common domain. Arch. Biochem. Biophys. 245, 483-493 (1986).

23. Hartl, F. U. Molecular chaperones in cellular protein folding. Nature 381, 571-579 (1996).

24. Fenton, W. A. \& Horwich, A. L. GroEL-mediated protein folding. Protein Sci. 6, 743-760 (1997).

Acknowledgements. We thank K. Furtak and J. Sutin for technical assistance, and D. Boisvert for discussion. This work was supported by grants from the NIH. S.G.B. is a fellow of the Wellcome Trust. yale.edu).

\section{DNA renaturation activity of the SMC complex implicated in chromosome condensation}

\author{
Takashi Sutani \& Mitsuhiro Yanagida \\ Department of Biophysics, Faculty of Science, Kyoto University, \\ Kitashirakawa-Oiwake, Sakyo-ku, Kyoto 606, Japan
}

Chromosome condensation occurs in mitosis before the separation of sister chromatids, and requires DNA topoisomerase II (refs 1,2$)$ and a group of proteins called $\mathrm{SMCs}^{3-5}$. The resulting condensed chromosomes in metaphase have a complex hierarchical structure ${ }^{6,7}$. SMCs, the components of condensed chromosomes, are also required for the separation of sister chromatids and gene dosage compensation, and are found in a range of organisms from yeasts to mammals ${ }^{8-13}$. However, the mechanisms by which the SMCs contribute to chromosome condensation are unknown. We have studied chromosomes in fission-yeast SMC mutants cut3-477 and cut14-208 (ref. 9), which remain largely non-condensed during mitosis at the restrictive temperature $\left(36^{\circ} \mathrm{C}\right)^{9}$. To test their role in DNA condensation, we isolated the proteins Cut 3 and Cut14 as an oligomeric complex, and tested their interactions with isolated DNA. The complex efficiently promoted the DNA renaturation reactions (the winding up of single-strand DNAs into double helical DNA) as much as $\sim 70$-fold more efficiently than $\operatorname{Rec}^{14}$, which is a bacterial protein with similar activity. The activity of the mutant complex was heat sensitive. As DNA winding by renaturation is a potential cause of supercoiling, the SMC complex may be implicated in promoting the higher-order DNA coiling found in condensed chromosomes.

To obtain isolated Cut3 and Cut14 proteins from Schizosaccharomyces pombe cells, six-histidine tags were attached and they were simultaneously overproduced using the inducible promoter REP1 (ref. 15) (Fig. 1a). Cells were collected and lysed, and the extract put over a Ni-NTA column ${ }^{16}$. Fractions containing Cut14-HA6His (relative molecular mass $\left.137,000\left(M_{\mathrm{r}} 137 \mathrm{~K}\right)\right)$ and Cut3 $(155 \mathrm{~K})$ at $80 \%$ purity were detected, indicating that the two proteins formed a complex ${ }^{10}$ in overproduced cells (Fig. 1b, c). The complex was further purified by phosphocellulose P11.

After sucrose-gradient centrifugation ${ }^{17}$, a broad distribution of Cut3-Cut14 at equal proportions was obtained at around 7-13S (the peak was at 13S; Fig. 1d, top). Gel filtration showed that the complex had an $M_{\mathrm{r}}$ of $600 \mathrm{~K}$ or larger (data not shown). The purified Cut3-Cut14 complex thus seemed to exist as a heterotetramer or possibly a larger complex, but a smaller form (heterodimer) might also exist. A similar oligomeric complex was also isolated from extracts of cells that were not overproduced (Fig. 1d, bottom).

The purified complex was found to renature DNA extremely efficiently (Fig. 2). Denatured, single-stranded linear DNAs were mixed with the complex, proteins were extracted, and the resulting 